\title{
Improved development by Taxol pretreatment after vitrification of in vitro matured porcine oocytes
}

\author{
Wen-Qing Shi ${ }^{1,2}$, Shi-En Zhu ${ }^{1,2}$, Dong Zhang ${ }^{3}$, Wei-Hua Wang ${ }^{4}$, Guo-Liang Tang ${ }^{1}$, \\ Yun-Peng $\mathrm{Hou}^{1,2}$ and Shu-Jun Tian ${ }^{1}$ \\ ${ }^{1}$ Laboratory of Animal Embryonic Biotechnology, College of Animal Science, China Agricultural University; \\ No. 2 Yuanmingyuan West Road, Haidian District, Beijing 100094, PR China, ${ }^{2}$ State Key Laboratories for \\ Agrobiotechnology, China Agricultural University; No.2 Yuanmingyuan West Road, Haidian District, Beijing \\ 100094, PR China, ${ }^{3}$ Department of Physiology and Biophysics, 223 Ullmann Building, Albert Einstein College of \\ Medicine, Bronx, NY 10461, USA and ${ }^{4}$ IVF Laboratory of Tomball Regional Hospital, 605 Holderrieth, Tomball, \\ TX 77375, USA
}

Correspondence should be addressed to Shi-En Zhu; Email: zhushien@cau.edu.cn

\begin{abstract}
This study was designed to examine the effect of Taxol pretreatment on vitrification of porcine oocytes matured in vitro by an open pulled straw (OPS) method. In the first experiment, the effect of Taxol pretreatment and fluorescein diacetate (FDA) staining on parthenogenetic development of oocytes was evaluated. In the second experiment, viability, microtubule organization and embryo development of oocytes were assessed after oocytes were exposed to vitrification/warming solutions or after vitrification with or without Taxol pretreatment. The results showed that Taxol pretreatment and/or FDA staining did not negatively influence the oocyte's developmental competence after parthenogenetic activation. After being exposed to vitrification/warming solutions, the survival rate $(83.3 \%)$ of the oocytes was significantly $(P<0.05)$ reduced as compared with that in the control $(\mathbf{1 0 0} \%)$. Vitrification/warming procedures further reduced the survival rates of oocytes regardless of oocytes being treated with $(62.1 \%)$ or without $(53.8 \%)$ Taxol. The proportions of oocytes with normal spindle configuration were significantly reduced after the oocytes were exposed to vitrification/warming solutions $(38.5 \%)$ or after vitrification with $\mathbf{( 1 0 . 3 \% )}$ or without $(\mathbf{4 . 1} \%)$ Taxol pretreatment as compared with that in control $(\mathbf{7 6 . 8} \%)$. The rates of two-cell-stage $(5.6-53.2 \%)$ embryos at $48 \mathrm{~h}$ and blastocysts $(0-3.8 \%)$ at $144 \mathrm{~h}$ after activation were significantly reduced after exposure to vitrification/warming solutions or after vitrification as compared with control $(90.9 \%$ and $26.6 \%$ respectively). However, the proportion of vitrified oocytes developed to two-cell stage was significantly higher when oocytes were pretreated with $\mathbf{2 4 . 3 \% )}$ than without $(\mathbf{5 . 6} \%)$ Taxol. These results indicate that pretreatment of oocytes with Taxol before vitrification helps to reduce the damage induced by vitrification and is a potential way to improve the development of vitrified porcine oocytes.

Reproduction (2006) 131 795-804
\end{abstract}

\section{Introduction}

The development of cryopreservation technology in nonhuman mammalian oocytes can be applied to humanassisted reproductive technology, in which oocyte cryopreservation shows many advantages for infertility treatment (Arav et al. 2002). Recently, vitrification has been widely used to cryopreserve oocytes in many mammals (Kono et al. 1991, Arav \& Zeron 1997, Isachenko et al. 1998), including man (Kuleshova et al. 1999, Yoon et al. 2000). As vitrification does not require a programmed freezer and the technique itself is easy, safe and highly efficient, it will become an indispensable method in cryobiology in the future (Kuleshova \& Lopata 2002, Liebermann et al. 2002). Normal offspring have been produced from the vitrified mature (Kono et al. 1991, Hamano et al. 1992, Kuleshova et al. 1999, Yoon et al. 2000) and immature oocytes (Vieira et al. 2002). However, it has been found that vitrification of porcine oocytes (Rojas et al. 2004) is much more difficult than in other mammals, such as mice (Wood et al. 1993) and cattle (Vieira et al. 2002). Various vitrification methods have been used to cryopreserve porcine oocytes (Ahn et al. 2004, Dinnyes et al. 2004, Rojas et al. 2004), but live births from vitrified oocytes have not been reported. Rojas et al. (2004) compared the viability of immature and mature porcine oocytes vitrified in ethylene glycol (EG)based solution by open pulled straw (OPS) vitrification, and found that porcine metaphase II (MII) oocytes (2-4 layers of cumulus cells remain) had better resistance to 
vitrification than germinal vesicle (GV) stage oocytes. The authors also found that $10.4 \%$ of vitrified oocytes could develop to the two-cell stage after in vitro fertilization (IVF). Recently, the vitrification solution (VS) with both EG and dimethyl sulfoxide (DMSO) has been successfully used to vitrify human and other mammalian oocytes (Hyttel et al. 2000, Vieira et al. 2002). Le Gal \& Massip (1999) reviewed the literature and found that only the OPS method (Vajta et al. 1998) yielded approximately 3\% blastocysts in mouse oocyte vitrification. Chen et al. (2000) reported that OPS can preserve higher rates of normal meiotic spindle morphology $(78 \%)$ and chromosome distribution (87\%) than the conventional straw method. It is well known that the porcine oocyte is more sensitive to low temperature than other mammalian oocytes; thus, successful methods for vitrification of porcine oocytes have not been developed.

Taxol has been used in cryopreservation of embryos (Dobrinsky et al. 2000), and it has been found that Taxol pretreatment can improve developmental competence of vitrified mouse (Park et al. 2001) and human (Fuchinoue et al. 2004) oocytes. However, to our knowledge, no study has reported the effect of Taxol treatment of porcine oocytes during vitrification. Therefore, in the present study, we investigated whether porcine oocytes matured in vitro can be vitrified by OPS and whether Taxol pretreatment can improve the vitrification efficiency in porcine oocytes.

\section{Materials and Methods}

All chemicals for this study were purchased from SigmaAldrich (St Louis, MO, USA) unless stated otherwise.

\section{In vitro maturation of oocytes}

The collection and culture of porcine oocytes were based on the protocol reported by Wang et al. (2000). Briefly, ovaries were collected from prepubertal pigs at a local slaughterhouse and transported to the laboratory within $2 \mathrm{~h}$ in $0.9 \%(\mathrm{w} / \mathrm{v}) \mathrm{NaCl}$ solution containing $75 \mu \mathrm{g}$ penicillin $\mathrm{G} / \mathrm{ml}$ and $50 \mu \mathrm{g}$ streptomycin sulfate/ml maintained at $33-37^{\circ} \mathrm{C}$. The room was always air-conditioned at a temperature of $25 \pm 1{ }^{\circ} \mathrm{C}$. The oocytes were aspirated from medium-sized follicles $(3-6 \mathrm{~mm}$ in diameter) with a 20 gauge needle fixed to a $10 \mathrm{ml}$ disposable syringe. Cumulus-oocyte complexes (COCs) surrounded by a compact cumulus mass with evenly granulated cytoplasm were selected. The COCs were washed four times in Tyrode's lactate (TL)-Hepes $\left(\mathrm{Ca}^{2+}\right.$ free) medium with $0.01 \%(\mathrm{w} / \mathrm{v})$ polyvinyl alcohol (TLHepes-PVA), and washed three times with maturation medium: TCM-199 (Gibco, Invitrogen Corporation, Grand Island, NY, USA) supplemented with $0.57 \mathrm{mmol}$ cysteine/l, $10 \mathrm{ng}$ epidermal growth factor (EGF)/ml, 10 IU equine chorionic gonadotropin (CG)/ml, $10 \mathrm{IU}$ human (h) $\mathrm{CG} / \mathrm{ml}$ and $10 \%(\mathrm{v} / \mathrm{v})$ porcine follicle fluid.
A group of $20-25$ oocytes was cultured at $39^{\circ} \mathrm{C}$ in an atmosphere of $5 \% \mathrm{CO}_{2}$ in air and saturated humidity. The oocytes were freed of cumulus cells at $44 \mathrm{~h}$ after culture by pipetting in the TL-Hepes-PVA medium containing $0.02 \% \quad(\mathrm{w} / \mathrm{v})$ hyaluronidase. Cumulus-free oocytes with the first polar body and even-granulated cytoplasm were used in the following experiments.

\section{Vitrification and warming of oocytes}

OPS was made by the method described by Vajta et al. (1998) with some modifications. Briefly, the straws $(250 \mu \mathrm{l}$; IMV, L'Aigle, France) were heat-softened and pulled manually. The pulled straws $(2-3 \mathrm{~cm}$ in length) were cut at the tapered end with a blade to ensure an inner diameter of about $0.2 \mathrm{~mm}$ and a wall thickness of about $0.02 \mathrm{~mm}$, which were measured with a microforge. The VS was modified from EFS40 (Zhu et al. 1993), which contained 20\% (v/v) EG, 20\% (v/v) DMSO, $18 \%(\mathrm{w} / \mathrm{v})$ Ficoll (mol. wt 70000 ) and $0.3 \mathrm{~mol}$ sucrose solution/l in a modified PBS solution without $\mathrm{Ca}^{2+}$ and $\mathrm{Mg}^{2+}\left(\mathrm{m}-\mathrm{PBS}^{-}\right)$. The oocytes were equilibrated for $90 \mathrm{~s}$ in equilibration solution (ES), that is,

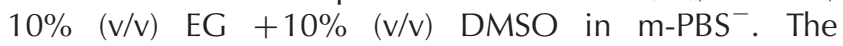
oocytes were loaded into the pulled end of straws by negative pressure and then exposed to VS for $30 \mathrm{~s}$ before the straws were plunged into liquid nitrogen (LN). The straws were stored in the LN for at least $24 \mathrm{~h}$ before warming. The warming solution (WS) was m$\mathrm{PBS}^{-}$containing various concentrations of sucrose. Stepwise warming was performed by placing the pulled end of the straws directly into $0.5 \mathrm{~mol}$ sucrose/l solution for $2 \mathrm{~min}$. The oocytes were then rinsed with other solutions of $0.5,0.25$ and $0.125 \mathrm{~mol}$ sucrose/l, each for $2 \mathrm{~min}$. All warming procedures were performed at $38.5^{\circ} \mathrm{C}$ on a warming stage fixed on the stereomicroscope, and the ambient atmosphere was always air-conditioned at a temperature of $25 \pm 1^{\circ} \mathrm{C}$.

\section{Assessment of viability and survival of oocytes}

For assessment of viability, oocytes exposed to different chemicals or vitrified were incubated for $30 \mathrm{~min}$ in a $\mathrm{CO}_{2}$ incubator, and then quickly washed three times in $\mathrm{m}-\mathrm{PBS}^{-}$solution. Finally, the oocytes were cultured in $\mathrm{m}-\mathrm{PBS}^{-}$containing $2.5 \mu \mathrm{g}$ fluorescein diacetate $(\mathrm{FDA}) / \mathrm{ml}$ for $3 \mathrm{~min}$ at $38.5^{\circ} \mathrm{C}$ and then washed three times in $\mathrm{m}-\mathrm{PBS}^{-}$solution before examination of membrane integrity under an inverted fluorescence microscope by the method of Zeron et al. (1999). High intensity of flourescence level was regarded as high viability, low fluorescence intensity was regarded as low viability, and nonfluorescence indicated dead oocytes. All images were acquired with a CCD video camera (DP70; Olympus, Tokyo, Japan) connected to the computer. Oocytes with high level of fluorescence and regular, 
spherical shape; without lysis; and not shrunken, swollen, or blackened were regarded as surviving.

\section{Labeling of oocytes for examination of microtubules (MT) and chromosomes}

The methods for examination of spindle morphology were based on a previous study (Sun et al. 2001) with some modifications. Briefly, cumulus-free oocytes were treated with $0.5 \%(\mathrm{v} / \mathrm{v})$ TritonX-100 in PHEM buffer $(60 \mathrm{mmol}$ Pipes/l, $25 \mathrm{mmol}$ Hepes/l, $10 \mathrm{mmol}$ EGTA/l, $4 \mathrm{mmol}$ $\mathrm{MgSO}_{4} / \mathrm{l}(\mathrm{pH} 7)$ ) for 5 min before being fixed in $4 \%$ formaldehyde in PHEM for 20 min. After being washed in PBS, the oocytes were blocked with 1\% BSA in PHEM for $1 \mathrm{~h}$ at room temperature and incubated in PHEM with $1 \%$ BSA containing monoclonal mouse antitubulin antibody (Jackson ImmunoResearch, West Grove, PA, USA) diluted 1:16000 overnight at $4^{\circ} \mathrm{C}$. The oocytes were washed four times in PBS with $0.05 \%$ Tween-20 (PBST) and then stained with fluorescein isothiocyanate (FITC)-conjugated goat antimouse IgG (Jackson ImmunoResearch) diluted 1:200 in PHEM with 1\% BSA for $45 \mathrm{~min}$. After four washes in PBST, the oocytes were stained with propidium iodide (PI) for chromosomes for $2 \mathrm{~min}$.

\section{Confocal microscopy of stained oocytes}

The oocytes were washed in PBST after staining and mounted with antifade solution $(0.5 \% n$-propyl gallate in $20 \mathrm{mmol}$ Tris/l, with $90 \%$ glycerol $(\mathrm{pH} 8)$ ) on the slides and examined by confocal laser scanning microscope (Zeiss LSM-510). Normal and abnormal morphologies were assessed by the former criterion (Liu et al. 2003). In brief, normal spindles show barrel-shaped morphology with the chromosomes aligned regularly at the metaphase plate and microtubules (MT) traversing the spindle from pole to pole or extending from the two poles to chromosomes; otherwise, those with unorganized, disassembled or absent MT were all classified as abnormal spindles. The chromosomes which aligned in a discrete bundle on the equator of the spindles were classified as normal chromosomes; otherwise, those with scattered or dispersed chromatids in the cytoplasm were all classified as abnormal chromosomes. Each experiment was repeated four times, and at least 30 oocytes were examined each time.

\section{Oocyte activation and embryo culture}

The oocytes were washed three times in TL-Hepes-PVA medium $\left(\mathrm{Ca}^{2+}\right.$-free) and then rinsed twice in the activation medium $\left(0.3 \mathrm{~mol}\right.$ mannitol $/ \mathrm{l}, 0.05 \mathrm{mmol} \mathrm{CaCl}_{2} / \mathrm{l}, 0.1 \mathrm{mmol}$ $\mathrm{MgCl}_{2} / \mathrm{l}$ and $\left.0.4 \%(\mathrm{~m} / \mathrm{v}) \mathrm{BSA}\right)$. The oocytes were transferred between two electrodes covered by activation medium in a chamber connected to an electrical pulsing machine (Fujihira Industry, Tokyo, Japan). The oocytes were stimulated with a direct current of $100 \mathrm{~V} / \mathrm{mm}$ for three consecutive $80-\mu$ s pulses at $100-\mathrm{ms}$ intervals. All oocytes activated were washed and cultured in North Carolina State University (NCSU)-23 medium (Petters \& Wells 1993) containing $7.5 \mu \mathrm{g}$ cytochalasin $\mathrm{B} / \mathrm{ml}$ (pre-equilibrated for $4-6 \mathrm{~h}$ in $5 \% \mathrm{CO}_{2}$ incubator). After treatment, the oocytes were completely washed, and each group of 20-25 oocytes cultured in NCSU-23 medium containing $0.4 \%(\mathrm{~m} / \mathrm{v})$ BSA (A-8022) in $100 \mu \mathrm{l}$ drops under mineral oil (pre-equilibrated for $4-6 \mathrm{~h}$ in $5 \% \mathrm{CO}_{2}$ incubator). Oocyte cleavage and blastocyst formation were examined and recorded at 48 and $144 \mathrm{~h}$ after activation respectively.

\section{Total nuclear counts}

Blastocysts were stained with $10 \mu \mathrm{g}$ bisbenzimide (Hoechst 33342 ) $/ \mathrm{ml}$ in NCSU-23 at $38.5^{\circ} \mathrm{C}$ for $10 \mathrm{~min}$ and then mounted and viewed under ultraviolet light on the same day. The total nuclear number of every blastocyst was counted by the number of nuclei under fluorescence microscopy.

\section{Experimental design}

In experiment 1, the effect of Taxol pretreatment and FDA staining on developmental competence of oocytes was tested. In vitro matured oocytes were randomly assigned to one of the following four experimental groups:

1. Control. No treatment was performed.

2. Taxol treatment. Oocytes were treated by $1 \mu \mathrm{mol}$ Taxol (T-7402)/l/m-PBS ${ }^{-}$for $30 \mathrm{~min}$ in the incubator.

3. FDA treatment. Oocytes were treated by $2.5 \mu \mathrm{g}$ $\mathrm{FDA} / \mathrm{ml}$ for $3 \mathrm{~min}$ on a warm stage $\left(38.5^{\circ} \mathrm{C}\right)$ without light.

4. Taxol plus FDA. Oocytes were stained by FDA after Taxol pretreatment. Cleavage rate, blastocyst rate and mean number of nuclei in blastocysts were examined after treatment and culture.

Table 1 Effect of taxol pretreatment and FDA staining on development of parthenogenetically activated porcine oocytes.

\begin{tabular}{lcccc}
\hline No. oocytes & Taxol pretreatment & FDA staining & No. cleaved $(\%)$ & No. blastocysts $(\%)$ \\
\hline 192 & - & - & $155(80.7 \pm 1.3)^{\mathrm{a}}$ & $48(25.0 \pm 2.8)^{\mathrm{a}}$ \\
201 & + & - & $171(85.2 \pm 1.7)^{\mathrm{a}}$ & $56(27.9 \pm 1.1)^{\mathrm{a}}$ \\
200 & - & + & $157(78.8 \pm 3.7)^{\mathrm{a}}$ & $46(23.1 \pm 2.1)^{\mathrm{a}}$ \\
196 & + & + & $160(81.6 \pm 1.3)^{\mathrm{a}}$ & $43(21.9 \pm 3.6)^{\mathrm{a}}$ \\
\hline
\end{tabular}

${ }^{a}$ Values with same superscript within same column are not significantly different at 0.05 level. 

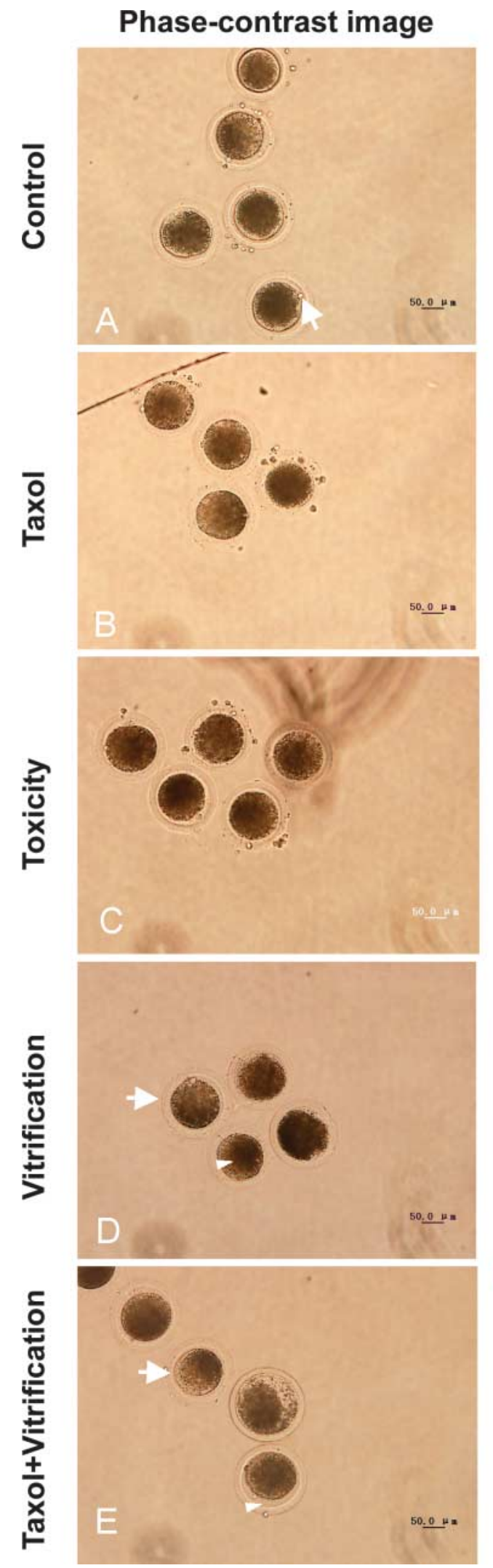

\section{Fluorescence image}
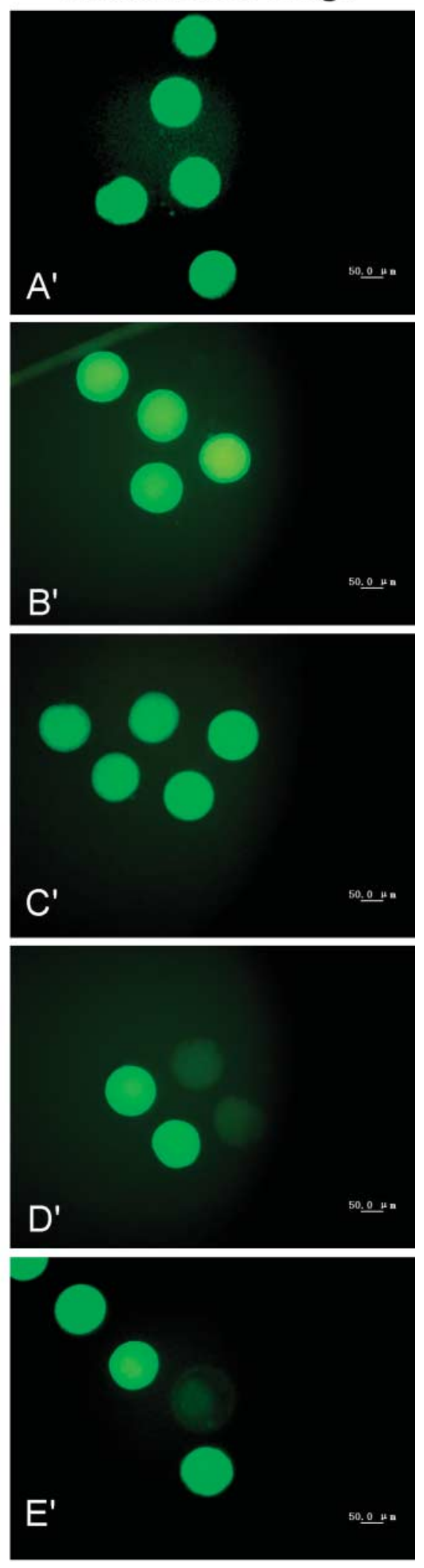

Figure 1 Phase-contrast and fluorescence microscope images of in vitro matured porcine oocytes exposed to vitrification solutions or Taxol, or after vitrification. Left column: phase-contrast microscope images; right column: fluorescence microscope images. $\left(A, A^{\prime}\right)$ Control group: oocytes were cultured in the medium without extra treatment. (B, $\mathrm{B}^{\prime}$ ) Toxicity-test group: oocytes were exposed to ES for $90 \mathrm{~s}$, VS for $30 \mathrm{~s}$ and WS $(0.5,0.25$ and $0.125 \mathrm{~mol}$ sucrose $/ \mathrm{l} / \mathrm{m}-\mathrm{PBS}^{-}$solutions), each for $2 \mathrm{~min}$. (C, $\mathrm{C}^{\prime}$ ) Taxol group: oocytes were cultured in $1 \mu \mathrm{mol} \mathrm{Taxol} / / / \mathrm{m} \mathrm{PBS}^{-}$for $30 \mathrm{~min}$ at $39^{\circ} \mathrm{C}$. (D, $\left.\mathrm{D}^{\prime}\right)$ Vitrification group: oocytes were vitrified- warmed. $\left(E, E^{\prime}\right)$ Taxol plus vitrification: oocytes were pretreated with $1 \mu \mathrm{mol}$ Taxol/l for $30 \mathrm{~min}$ at $39^{\circ} \mathrm{C}$ and then vitrifiedwarmed. Oocyte viability was indicated by intense fluorescence; nonfluorescence indicated nonviability. Bar $=50 \mu \mathrm{m}$. Arrow in panel A indicates first polar body; Arrow in panel D indicates unsmooth Zona Pellucida; Arrow head in panel D indicates very dark cytoplasm; Arrow in panel $\mathrm{E}$ indicates uneven cytoplasm; Arrow head in panel $\mathrm{E}$ indicates bigger perivitelline space. 
Table 2 Effect of different treatment on spindle morphology and chromosomes alignment in porcine oocytes.

\begin{tabular}{|c|c|c|c|}
\hline \multirow[b]{2}{*}{ Groups } & \multirow[b]{2}{*}{ No. examined } & \multicolumn{2}{|c|}{ No. $(\%)$ of oocytes with } \\
\hline & & Normal spindle configuration & Normal chromosome alignment \\
\hline Control & 124 & $95(76.8 \pm 1.27)^{\mathrm{a}}$ & $119(81.6 \pm 1.70)^{\mathrm{a}}$ \\
\hline Toxicity & 115 & $45(38.5 \pm 5.02)^{b}$ & $84(73.5 \pm 6.80)^{\mathrm{a}}$ \\
\hline Vitrification & 122 & $5(4.1 \pm 0.80)^{\mathrm{C}}$ & $68(55.6 \pm 5.75)^{\mathrm{b}}$ \\
\hline Taxol + vitrification & 116 & $12(10.3 \pm 1.27)^{\mathrm{d}}$ & $81(68.1 \pm 1.58)^{a b}$ \\
\hline
\end{tabular}

abcd Values with different superscripts within same column are significantly different at 0.05 level.

More than 40 oocytes in very group were examined each time, and experiments were repeated four times.

In experiment 2, the effect of different treatments and vitrification on oocyte survival, MT and chromosomes, and developmental competence was examined. In vitro matured oocytes were randomly classed into the following four groups:

1. Control. No treatment was performed.

2. Toxicity test of VS/WS. Oocytes were exposed to ES for $90 \mathrm{~s}$ and VS for $30 \mathrm{~s}$, and then rinsed in WS (0.5, 0.25 and $0.125 \mathrm{~mol}$ sucrose $/ \mathrm{l} / \mathrm{m}-\mathrm{PBS}^{-}$solution), each for $2 \mathrm{~min}$.

3. Vitrification. Oocytes were vitrified and warmed as in the OPS vitrification procedure mentioned above.

4. Taxol plus vitrification. Oocytes were pretreated with $1 \mu \mathrm{mol}$ Taxol/l for $30 \mathrm{~min}$ at $39^{\circ} \mathrm{C}$ and vitrifiedwarmed as in the OPS vitrification procedure.

After treatment and/or vitrification/warming, half of the oocytes from each group were fixed and labeled for examination of MT and chromosomes by confocal microscopy. Another half of the oocytes were examined for viability and survival. Only survival oocytes were further activated, and their parthenogenetic developmental competence was assessed by cleavage and blastocyst development.

\section{Statistical analyses}

All data are presented as the mean \pm S.E.M. All percentage data were subjected to arcsine transformation before statistical analysis. Data were analyzed by one-way ANOVA by Duncan's test. A value of $P<0.05$ was considered statistically significant.

\section{Results}

\section{Experiment 1}

\section{Effect of Taxol and FDA pretreatment}

As shown in Table 1, the developmental competence of MII oocytes after Taxol pretreatment or FDA staining was the same as that in the control, showing no statistical differences $(P>0.05)$ in the cleavage rates $(78.8-85.2 \%)$, blastocyst formation rates $(21.9-27.9 \%)$ and mean numbers (24.0-26.2) of nuclei in the blastocysts.

\section{Experiment 2}

FDA staining and survival assessment of oocytes

Figure 1 shows normal phase-contrast images (panels $A-E$ ) and fluorescence images (panels $A^{\prime}-E^{\prime}$ ) of oocytes. Only cytoplasm was stained green by FDA. After treatment and short-time culture, the oocytes exposed to VS/WS could recover normal shape (Fig. $1 \mathrm{~B}$ and $\mathrm{C}$ ), and their relative viability was the same as control oocytes. However, after vitrification and warming procedures, some oocytes did not recover normal morphology (Fig. 1D and E). These oocytes were considered as nonsurviving: the cytoplasm was very dark (Fig. 1D, arrowhead) or uneven (Fig. 1E, arrow), or the zona pellucida was not smooth (Fig. 1D, arrow) or swollen (Fig. 1D, arrowhead).

\section{Chromosomes and spindle configuration of oocytes after treatment}

As shown in Table 2, most oocytes had normal spindle configuration $(76.8 \%)$ and chromosome alignment $(81.6 \%)$ in the control. Toxicity treatment with VS/WS reduced the rate of normal spindle configuration to $38.5 \%$, but had little influence on the rate $(73.5 \%)$ of normal chromosome alignment in the oocytes. After vitrification and warming, however, $4.1-10.3 \%$ of oocytes had normal spindle configuration, and $55.6-68.1 \%$ of oocytes had normal chromosome alignment (Fig. 2). When Taxol was used before vitrification, the rate of oocytes with normal spindles was significantly $(P<0.05)$ higher $(10.3 \%)$ than with vitrification without Taxol pretreatment (4.1\%).

\section{Survival and development of the treated oocytes}

Survival rates of oocytes after treatment are shown in Table 3. After exposure to VS/WS, most of the oocytes survived, although the survival rate $(83.3 \%)$ was lower than that in the control (100\%). However, 62.1\% (Taxol plus vitrification) and $53.8 \%$ (vitrification) of the oocytes survived after vitrification and warming for $30 \mathrm{~min}$, rates that were significantly $(P<0.05)$ lower than those in control and toxicity groups.

As shown in Table 3, 90.9\% of the control oocytes developed to two-cell stage after 48-h culture and $26.6 \%$ reached blastocyst stage after 144-h culture. However, in the toxicity-tested group, both cleavage rate and blastocyst formation rate were reduced significantly $(53.2 \%$ and 

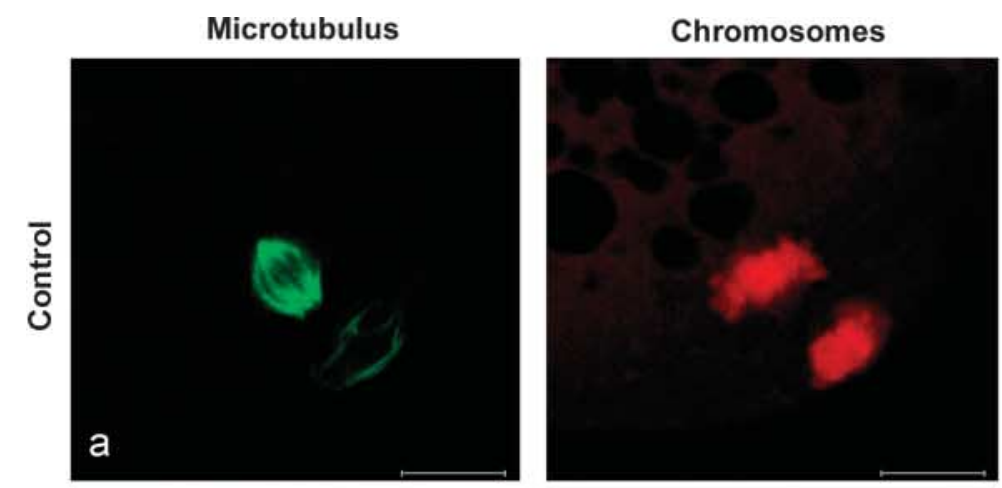

Microtubulus + chromosomes
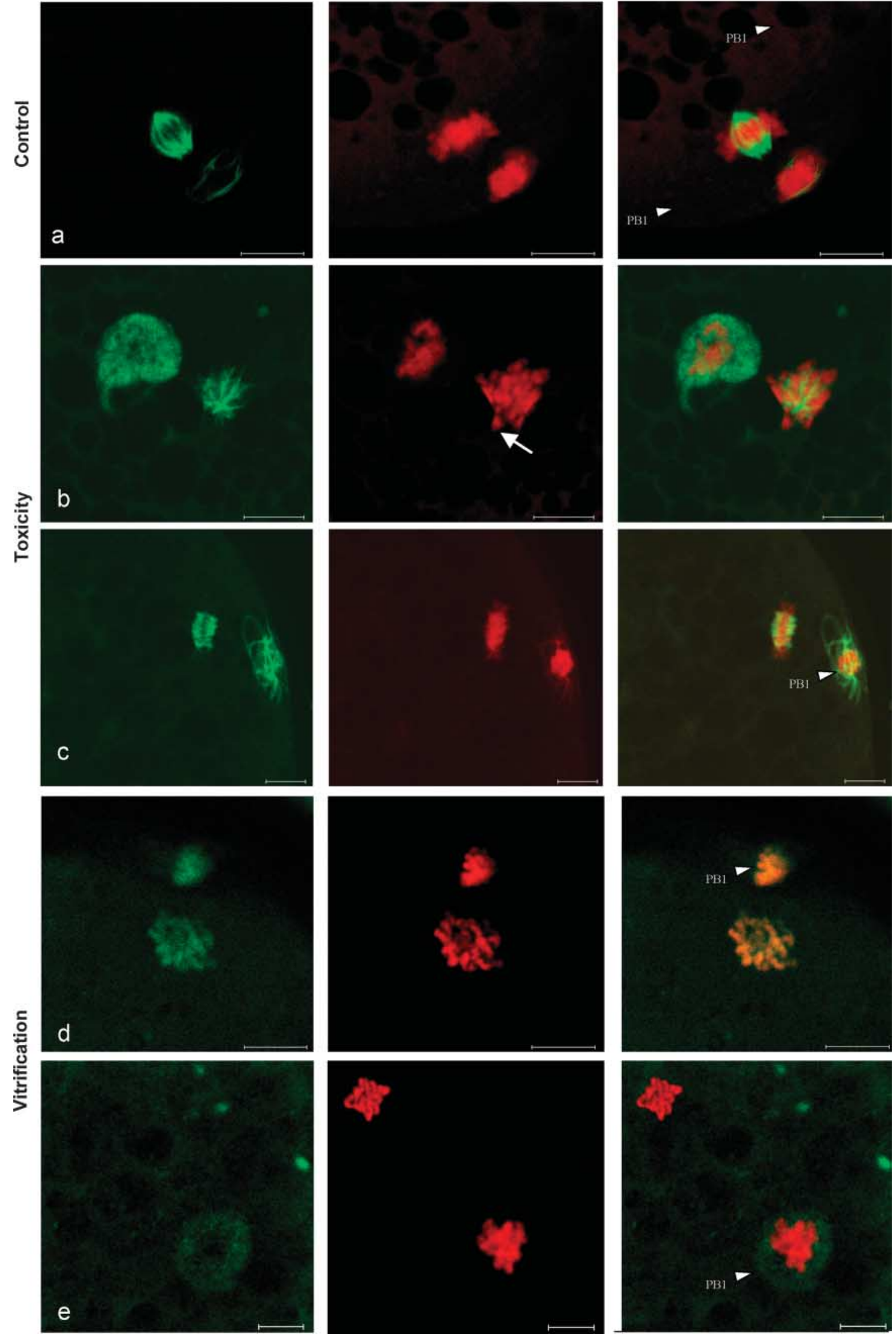

Figure 2 (Continued) 

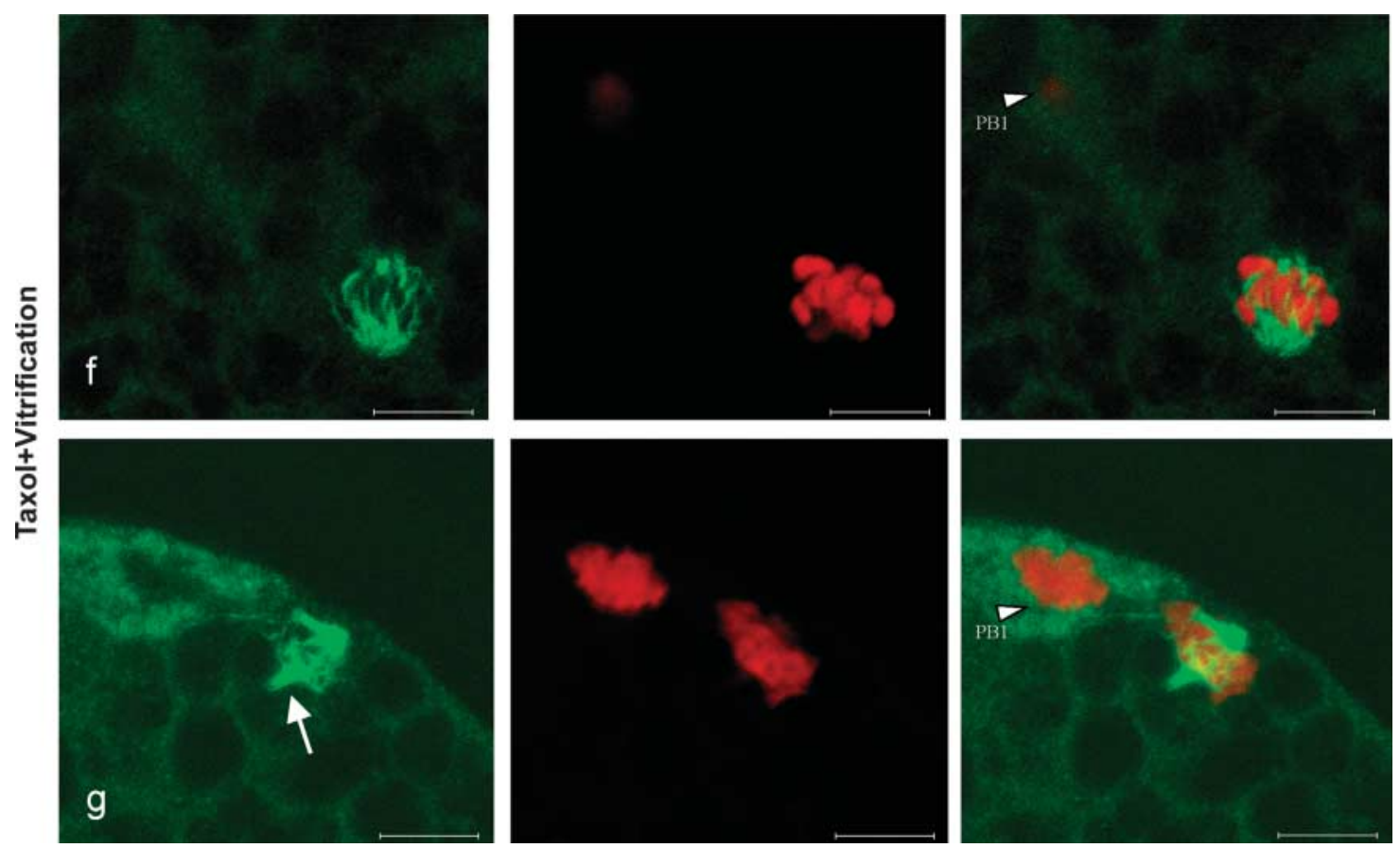

Figure 2 Confocal micrographs of spindles and chromosomes in the porcine oocytes exposed to vitrification solution or after vitrification. Panel (a) shows an oocyte (without treatment) with normal spindle configuration, indicating a barrel-shaped structure and the chromosomes arranged on the equator of the metaphase plate. Panels (b) and (c) show oocytes exposed to ES-VS and WS with abnormal spindle, with some chromosomes displaced from the metaphase plate (b, arrow), and an oocyte with a normal spindle and chromosomal organization. Panels (d) and (e) show oocytes vitrified without taxol pretreatment. The spindles are obviously disorganized (d) or almost completely disassembled (e). The chromosomes are dispersed and less condensed. Panels $(\mathrm{f})$ and $(\mathrm{g})$ show oocytes vitrified after taxol pretreatment. The spindle is obvious with rounded poles and the chromosomes arranged on the plate of the equator but condensed ( $\mathrm{f}$ ), or microtubules from one pole to another are assembled into big bundles (arrow) and the spindle shows pointed poles but normal chromosomes (g). Green images represent microtubules, red images represent chromosomes, and yellow images represent the merging of green and red images. Arrowheads indicate the first polar body (PB1). Bar $=10 \mu \mathrm{m}$.

$3.8 \%$ respectively) as compared with control $(P<0.05)$. Vitrification further reduced both cleavage and blastocyst formation (5.6-24.3\% and $0 \%$ respectively). The cleavage rates of oocytes vitrified by Taxol pretreatment $(24.3 \%)$ was higher $(P<0.05)$ than that without Taxol pretreatment (5.6\%), although all two-cell embryos did not develop to blastocyst stage.

\section{Discussion}

The present study indicates that porcine oocytes matured in vitro can be vitrified by the OPS method, and pretreatment of oocytes with Taxol significantly improved the developmental competence of oocyte after vitrification.

Our present study also indicates that Taxol treatment itself does not affect oocyte quality, which has been assessed by the blastocyst development of parthenogenetically activated oocytes. The viability or survival of oocytes was affected by both VS/WS and vitrification/warming procedures. FDA has been previously used to assess the viability of mouse embryos (Mohr \& Trounson 1980), porcine GV oocytes (Didion et al. 1990) and bovine oocytes (Zeron et al. 1999). Oocyte viability was evidenced by intense fluorescence; nonfluorescence indicated nonviability. Therefore, FDA staining is useful and more objective in survival assessment. In the present experiment, the validity of MII porcine oocytes was proved by FDA staining.

Cryopreservation always induces oocyte damage. Cryodamage to oocytes is caused by mechanical, thermal or chemical factors. Vitrification can prevent the formation of intracellular ice crystals that may cause mechanical damage (Rall \& Fahy 1985). In the present study, the inner diameter of the pulled straws allows just a porcine oocyte (without cumulus cell) to go through by negative pressure, and the volume of VS can be limited $(\sim 1 \mu \mathrm{l}$ VS in the pulled straw) so that the cooling rate can be increased. By this method, intracellular cryodamage to oocytes can be avoided or minimized. However, our results showed that porcine oocytes suffer irreversible damage after vitrification, especially on the meiotic spindle configuration. Furthermore, we found that spindles were also damaged when the oocytes were treated by EG and DMSO only, indicating that the cooling rate is not the only key factor to affect oocyte viability during vitrification. Further studies must determine how to optimize the vitrification procedure and modify the VS in order to achieve higher 
survival rates after vitrification of porcine oocytes. These results also suggest that injury to the oocyte cytoskeleton is an important cryoinjury during vitrification.

Chemicals in the VS are the major factors that affect oocyte survival rate during vitrification. Oocytes exposed to cryoprotective agents (CPAs) could depolymerize MT (Johnson \& Pickering 1987). This depends on the concentration of CPAs. Chen et al. (2000) found that the spindles in mouse oocytes became disorganized or disappeared when they were exposed to VS containing $5.5 \mathrm{~mol}$ EG/l and $1.0 \mathrm{~mol}$ sucrose/l. In the present experiment, when oocytes were exposed to ES-VS and WS, their developmental competence was significantly reduced, suggesting that these chemicals, and their concentrations and exposing time are not suitable for porcine oocyte vitrification under the present experimental conditions, and that further studies are necessary.

The survival rate of vitrified oocytes is not sufficient to evaluate potential quality. Testing the developmental competence of oocytes is indispensable to examine their viability. Because of the high rate of polyspermy in in vitro fertilized pig oocytes (Nagai 1994), artificial activation, especially electrical activation of oocytes, becomes a useful approach to test porcine oocyte developmental competence. The electrical activation method in porcine oocytes has proved to be effective and has been used in somatic nuclear transfer in pig (Onishi et al. 2000, Polejaeva et al. 2000); 25-26.6\% of oocytes developed into blastocysts in our control experiments. However, vitrification and exposing the oocytes to VS/WS dramatically reduced oocyte development, indicating that vitrified porcine oocytes have very low viability even if they survive.

In the present study, our results indicate that low concentrations of Taxol did not reduce the development of parthenogenetically activated porcine oocytes. Taxol can lower the critical tubulin concentration in vitro for assembly and promote tubulin to reassembly in vitro (Schiff et al. 1979, De Brabander et al. 1981), and it can be used to investigate the system of microtubule assembly/disassembly in pig oocytes at different developmental stages (Sun et al. 2001). MT in porcine oocytes are vulnerable to cryoinjury in vitro (Didion et al. 1990, Wang et al. 2001, Liu et al. 2003). Morphology of MT and microfilaments in porcine oocytes can resume after 1-h culture, after oocytes are thawed or warmed, and the distribution of MT and microfilaments recovers, but low temperature leads to distinct impairment of the fertilization of oocytes and the growth of embryos (Wu et al. 1999). Moreover, cryopreservation can affect configuration of MT, block the formation of the first meiotic spindle (Rojas et al. 2004), and lead to cell death or abnormal development (Shaw et al. 2000). In the present experiment, the cleavage rate of oocytes vitrified by OPS increased from $5.6 \%$ to $24.3 \%$ by pretreatment of Taxol, the survival rates of porcine MII oocytes vitrified with Taxol pretreatment increased from $53.9 \%$ to $62.1 \%$, and normal spindle configuration rates increased from $4.1 \%$ to $10.3 \%$. From these results, it can be concluded that Taxol pretreatment of oocytes before vitrification significantly improves the parthenogenetic development of oocytes after subsequent vitrification and warming. These results also indicate that stabilization of the MT in the oocytes may reduce the damage induced by vitrification.

Chen et al. (2000) examined the intracellular cryodamage of mouse oocytes by immunofluorescence staining of spindles and found that 1-3-h incubation after thawing can significantly increase rates of oocytes with normal spindles. In the present study, rates of oocytes with normal spindles remained low after 30-min incubation. It is still unclear whether normal cytoskeleton configuration in porcine oocytes can be increased by longer incubation.

Partial removal of lipid from porcine embryos (Nagashima et al. 1995) and oocytes (Nagashima et al. 1999) also reduces sensitivity to chilling and improves development after vitrification. There are abundant cytoplasmic lipids in porcine oocytes, and the amount of triglyceride, the main component of the lipids, is threefold higher than that in cattle and sheep oocytes (McEvoy et al. 2000). These lipids may play a significant role in cell structure and function, especially in biological membranes, but it is unclear why pig oocytes contain so much lipid (Sturmey \& Leese 2003). The maturation of vitrified immature porcine oocytes can be improved (from $3.7 \%$ to $15.0 \%$ ) by partial removal of cytoplasmic lipids (Park et al. 2005). The effect of delipidization on oocyte vitrification has been reported (Nagashima et al. 1999), and it was found that about $56 \%$ of the oocytes vitrified after delipidization survived, and $6 \%$ of oocytes could develop to morula stage after fertilization by subzonal sperm injection.

In conclusion, we found that both exposure to VS/WS and vitrification/warming procedures can cause spindle

Table 3 Effect of different treatments on development of parthenogenetically activated oocytes.

No. (\%) of oocytes developed to

\begin{tabular}{|c|c|c|c|c|}
\hline \multirow[b]{2}{*}{ Groups } & \multirow[b]{2}{*}{ No. of oocytes examined } & \multirow[b]{2}{*}{ No. $(\%)$ of surviving oocytes } & \\
\hline & & & Two-cell stage & Blastocyst stage \\
\hline Control & 122 & $122(100 \pm 0)^{\mathrm{a}}$ & $112(90.9 \pm 3.59)^{\mathrm{a}}$ & $32(26.6 \pm 2.65)^{a}$ \\
\hline Toxicity & 138 & $115(83.3 \pm 3.48)^{b}$ & $72(53.2 \pm 10.36)^{b}$ & $5(3.8 \pm 2.01)^{b}$ \\
\hline Vitrification & 158 & $85(53.8 \pm 5.62)^{\mathrm{C}}$ & $9(5.6 \pm 0.79)^{\mathrm{c}}$ & $0^{\mathrm{b}}$ \\
\hline Taxol + vitrification & 132 & $82(62.1 \pm 1.14)^{\mathrm{C}}$ & $32(24.3 \pm 2.78)^{d}$ & $0^{\mathrm{b}}$ \\
\hline
\end{tabular}

abc Values with different superscripts within same column are significantly different at 0.05 level. 
disassembly and chromosome misalignment, reducing the survival and developmental competence in porcine oocytes. Taxol pretreatment can reduce the damage induced by vitrification, thus improving the development of porcine oocytes after vitrification. As no blastocyst development was obtained after the oocytes were vitrified under the present conditions, further studies are necessary to optimize the VS/WS or vitrification/warming procedures.

\section{Acknowledgements}

This work was supported by a grant from National ' 863 ' Project Foundation of China (No. 2004AA213070) and from the National Natural Science Foundation of China (no. 30571334). We thank Dr Meng QingGang for proofreading the manuscript, and Du MeiHong, Pan HongMei and Ren $\mathrm{Yi}_{i}$ for their assistance in the laboratory. The authors declare that there is no conflict of interest that would prejudice the impartiality of this scientific work.

\section{References}

Ahn MH, Seok HB, Kim ID \& Son DS 2004 Comparison of the survival of in vitro-derived porcine oocytes and embryos vitrified by open pulled straw, electron microscope grid, and nylon loop system. Reproduction, Fertility, and Development 16 162-163.

Arav A, Yavin S, Zeron Y, Natan D, Dekel I \& Gacitua H 2002 New trends in gamete's cryopreservation. Molecular and Cellular Endocrinology $18777-81$.

Arav A \& Zeron Y 1997 Vitrification of bovine oocytes using modified minimum drop size technique (MDS) is effected by the composition and concentration of the vitrification solution and by the cooling conditions. Theriogenology 47 341-342.

Chen SU, Lien YR, Chen HF, Chao KH, Ho HN \& Yang YS 2000 Open pulled straws for vitrification of mature mouse oocytes preserve patterns of meiotic spindles and chromosomes better than conventional straws. Human Reproduction 15 2598-2603.

De Brabander M, Geuens G, Nuydens R, Willebrords R \& De Mey J 1981 Taxol induces the assembly of free microtubules in living cells and blocks the organizing capacity of the centrosomes and kinetochores. PNAS 78 5608-5612.

Didion BA, Pomp D, Martin MJ, Homanics GE \& Markert CL 1990 Observations on the cooling and cryopreservation of pig oocytes at the germinal vesicle stage. Journal of Animal Science $\mathbf{6 8}$ 2803-2810.

Dinnyes A, Dai Y, Jiang S \& Yang X 2004 Cryopreservation of in vitro porcine oocytes by solid surface vitrification. Reproduction, Fertility, and Development 17 191-192.

Dobrinsky JR, Pursel VG, Long CR \& Johnson LA 2000 Birth of piglets after transfer of embryos cryopreserved by cytoskeletal stabilization and vitrification. Biology of Reproduction $62564-570$.

Fuchinoue K, Fukunaga N, Chiba S, Nakajo Y, Yagi A \& Kyono K 2004 Freezing of human immature oocytes using cryoloops with Taxol in the vitrification solution. Journal of Assisted Reproduction and Genetics 21 307-309.

Hamano S, Koikeda A, Kuwayama M \& Nagai T 1992 Full-term development of in vitro-matured, vitrified and fertilized bovine oocytes. Theriogenology 38 1085-1090.

Hyttel P, Vajta G \& Callesen H 2000 Vitrification of bovine oocytes with the open pulled straw method: ultrastructural consequences. Molecular Reproduction and Development 56 80-88.

Isachenko V, Soler C, Isachenko E, Perez-Sanchez F \& Grishchenko V 1998 Vitrification of immature porcine oocytes: effects of lipid droplets, temperature, cytoskeleton, and addition and removal of cryoprotectant. Cryobiology 36 250-253.
Johnson MH \& Pickering SJ 1987 The effect of dimethylsulphoxide on the microtubular system of the mouse oocyte. Development $100313-324$.

Kono T, Kwon OY \& Nakahara T 1991 Development of vitrified mouse oocytes after IVF. Cryobiology 28 50-54.

Kuleshova L, Gianaroli L, Magli C, Ferraretti A \& Trounson A 1999 Birth following vitrification of a small number of human oocytes: case report. Human Reproduction 14 3077-3079.

Kuleshova LL \& Lopata A 2002 Vitrification can be more favorable than slow cooling. Fertility and Sterility 78 449-454.

Le Gal F \& Massip A 1999 Cryopreservation of cattle oocytes: effects of meiotic stage, cycloheximide treatment, and vitrification procedure. Cryobiology 38 290-300.

Liebermann J, Nawroth F, Isachenko V, Isachenko E, Rahimi G \& Tucker M 2002 Potential importance of vitrification in reproductive medicine. Biology of Reproduction 67 1671-1680.

Liu RH, Sun QY, Li YH, Jiao LH \& Wang WH 2003 Effects of cooling on meiotic spindle structure and chromosome alignment within in vitro matured porcine oocytes. Molecular Reproduction and Development 65 212-218.

McEvoy TG, Coull GD, Broadbent PJ, Hutchinson JS \& Speake BK 2000 Fatty acid composition of lipids in immature cattle, pig and sheep oocytes with intact zona pellucida. Journal of Reproduction and Fertility $118163-170$.

Mohr LR \& Trounson AO 1980 The use of fluorescein diacetate to assess embryo viability in the mouse. Journal of Reproduction and Fertility $\mathbf{5 8}$ 189-196.

Nagai T 1994 Current status and perspectives in IVM-IVF of porcine oocytes. Theriogenology 41 73-78.

Nagashima H, Kashiwazaki N, Ashman RJ, Grupen CG \& Nottle MB 1995 Cryopreservation of porcine embryos. Nature $\mathbf{3 7 4} 416$.

Nagashima H, Cameron RDA, Kuwayama M, Young M, Beebe L, Blackshaw AW \& Nottle MB 1999 Survival of porcine delipated oocytes and embryos after cryopreservation by freezing or vitrification. Journal of Reproduction and Development 45 167-176.

Onishi A, Iwamoto M, Akita T, Mikawa S, Takeda K, Awata T, Hanada H \& Perry ACF 2000 Pig cloning by microinjection of fetal fibroblast nuclei. Science 289 1188-1190.

Park KE, Kwon IK, Han MS \& Niwa K 2005 Effects of partial removal of cytoplasmic lipid on survival of vitrified germinal vesicle stage pig oocytes. Journal of Reproduction and Development 51 151-160.

Park SE, Chung HM, Cha KY, Hwang WS, Lee ES \& Lim JM 2001 Cryopreservation of ICR mouse oocytes: improved post-thawed preimplantation development after vitrification using Taxol, a cytoskeleton stabilizer. Fertility and Sterility 75 1177-1184.

Petters RM \& Wells KD 1993 Culture of pig embryos. Journal of Reproduction and Fertility 48 Suppl 61-73.

Polejaeva IA, Chen SH, Vaught TD, Page RL, Mullins J, Ball S, Dai Y, Boone J, Walker S, Ayares DL, Colman A \& Campbell KH 2000 Cloned pigs produced by nuclear transfer from adult somatic cells. Nature 407 86-90.

Rall WF \& Fahy GM 1985 Ice-free cryopreservation of mouse embryos at $-196^{\circ} \mathrm{C}$ by vitrification. Nature $313573-575$.

Rojas C, Palomo MJ, Albarracin JL \& Mogas T 2004 Vitrification of immature and in vitro matured pig oocytes: study of distribution of chromosomes, microtubules, and actin microfilaments. Cryobiology 49 211-220.

Schiff PB, Fant J \& Horwitz SB 1979 Promotion of microtubule assembly in vitro by taxol. Nature 277 665-667.

Shaw JM, Oranratnachai A \& Trounson AO 2000 Fundamental cryobiology of mammalian oocytes and ovarian tissue. Theriogenology $5359-72$.

Sturmey RG \& Leese HJ 2003 Energy metabolism in pig oocytes and early embryos. Reproduction 126 197-204.

Sun QY, Lai L, Wu GM, Park KW, Day BN, Prather RS \& Schatten H 2001 Microtubule assembly after treatment of pig oocytes with taxol: correlation with chromosomes, $\gamma$-tubulin, and MAP kinase. Molecular Reproduction and Development 60 481-490. 
Vajta G, Holm P, Kuwayama M, Booth PJ, Jacobsen H, Greve T, Callesen H \& Callesent H 1998 A new way to avoid cryoinjuries of mammalian ova and embryos: the OPS vitrification. Molecular Reproduction and Development 51 53-58.

Vieira AD, Mezzalira A, Barbieri DP, Lehmkuhl RC, Rubin MI \& Vajta G 2002 Calves born after open pulled straw vitrification of immature bovine oocytes. Cryobiology 45 91-94.

Wang WH, Abeydeera LR, Prather RS \& Day BN 2000 Polymerization of nonfilamentous actin into microfilaments is an important process for porcine oocyte maturation and early embryo development. Biology of Reproduction 62 1177-1183.

Wang WH, Meng L, Hackett RJ, Odenbourg R \& Keefe DL 2001 Limited recovery of meiotic spindles in living human oocytes after cooling-rewarming observed using polarized light microscopy. Human Reproduction 16 2374-2378.

Wood MJ, Barros C, Candy C, Carroll CJ, Melendez J \& Whittingham DG 1993 High rates of survivals and fertilization of mouse and hamster oocytes after vitrification in dimethysulphoxide. Biology of Reproduction 49 489-495.

Wu B, Tong J \& Leibo SP 1999 Effects of cooling germinal vesicle-stage bovine oocytes on meiotic spindle formation following in vitro maturation. Molecular Reproduction and Development 54 388-395.
Yoon TK, Chung HM, Lim JM, Han SY, Ko IJ \& Cha KY 2000 Pregnancy and delivery of healthy infants developed from vitrified oocytes in a stimulated in vitro fertilization-embryo transfer program. Fertility and Sterility 74 180-181.

Zeron Y, Pearl M, Borochov A \& Arav A 1999 Kinetic and temporal factors influence chilling injury to germinal vesicle and mature bovine oocytes. Cryobiology 38 35-42.

Zhu SE, Kasai M, Otoge H, Sakurai T \& Machida T 1993 Cryopreservation of expanded mouse blastocysts by vitrification in ethylene glycol-based solutions. Journal of Reproduction and Fertility 98 139-145.

Received 20 July 2005

First decision 2 September 2005

Revised manuscript received 8 December 2005

Accepted 12 December 2005 